\title{
RISCOS OCUPACIONAIS NA ATIVIDADE DOS PROFISSIONAIS DE SAÚDE DA ATENÇÃo BÁSICA
}

\author{
Leilane Grazziela Nascimento Almeida Discentes do Curso de Especialização em \\ Enfermagem do Trabalho da Escola \\ Bahiana de Medicina e Saúde Pública
}

\author{
Samantha Coelho Torres Discentes do Curso de Especialização em \\ Enfermagem do Trabalho da Escola \\ Bahiana de Medicina e Saúde Pública
}

\author{
Cristiane Magali Freitas dos Santos Msc em Enfermagem pela Universidade \\ Federal da Bahia. Coordenadora do Curso \\ de Especialização de Enfermagem do \\ Trabalho e Docente da Graduação de \\ Enfermagem da Escola Bahiana de \\ Medicina e Saúde Pública
}

\begin{abstract}
Resumo
Objetivo: analisar os riscos ocupacionais a que estão expostos os profissionais atuantes na Atenção Básica de Saúde. Método: artigo de revisão a partir de um levantamento bibliográfico na base de dados da biblioteca virtual de saúde. Foram selecionados 20 artigos os quais foram analisados com base em literatura específica e na legislação referente à saúde do trabalhador. Os artigos foram classificados por categoria de risco: físicos, químicos, biológicos, ergonômicos, psicossociais e de acidentes. Resultados: os diferentes riscos ocupacionais foram abordados nos estudos de forma não concomitante e com nomenclatura diversificada, sendo o risco psicossocial o mais referido nos estudos. Observou-se que os profissionais atuantes na Atenção Básica estão expostos, além dos riscos inerentes às atividades em saúde, a outros riscos peculiares as características do trabalho neste nível de atenção e que muitos profissionais não possuem consciência dos riscos ocupacionais a que estão expostos. Conclusão: há um número limitado de trabalhos referentes a esta temática, ao que se faz necessário ampliar as discussões sobre os riscos ocupacionais, identificando as especificidades da atenção básica à saúde; fortalecer as ações de vigilância em saúde do trabalhador e rever a legislação que rege o setor a fim de proporcionar informações que levem os profissionais a refletirem sobre o seu auto-cuidado, bem como reivindicarem por melhores condições de trabalho.
\end{abstract}

Palavras-chave: Riscos ocupacionais; Atenção básica à saúde; Atenção primária à saúde; Saúde da família; Doenças ocupacionais.

\section{OCCUPATIONAL HAZARDS IN THE ACTIVITY OF BASIC CARE HEALTH PROFESSIONALS}

\begin{abstract}
Objective: analyze the occupational hazards faced by professionals working in Primary Health. Method: review article from a bibliographic database on the health virtual library. We selected 20 articles were analyzed based on relevant literature and legislation relating to workers' health. The articles were classified by risk category: physical, chemical, biological, ergonomic, psychosocial and accidents. Results: the different occupational hazards were addressed in the studies in a non-concurrent and diverse nomenclature, the risk psychosocial the most referred in the studies. Noted that the professionals working in Primary Care are exposed beyond the risks inherent to the activities in health, other risks peculiar characteristics of the work at this level of attention and that many professionals have no consciousness of occupational hazards they are exposed. Conclusion: there are a limited number of studies concerning this subject, it is necessary to expand the discussion of occupational hazards, identifying the specifics of the basic health care, strengthen the actions of the worker's health surveillance and review the legislation governing the sector to provide information leading practitioners to reflect on their self-care, as well as claim for better working conditions.
\end{abstract}


Keywords: Occupational hazards; Basic health care; Primary health care; Family health; Occupational diseases. RIESGOS LABORALES EN LA ACTIVIDAD DE LOS PROFESIONALES DE LA SALUD DE ATENCIÓN BÁSICA

\begin{abstract}
Resumen
Objetivo: analizar los riesgos laborales que enfrentan los profesionales que trabajan en Atención Primaria de la Salud. Método: artículo de revisión de una base de datos bibliográfica en la biblioteca virtual en salud. Hemos seleccionado 20 artículos fueron analizados sobre la base de la bibliografía pertinente y la legislación relativa a la salud de los trabajadores. Los artículos fueron clasificados por categoría de riesgo: físicos, químicos, biológicos, ergonómicos, psicosociales y accidentes. Resultados: los riesgos laborales diferentes se abordaron en los estudios en una nomenclatura no concurrentes y diversas, y el riesgo psicosocial lo más mencionado en los estudios. Se observó que los profesionales que trabajan en atención primaria están expuestos, más allá de los riesgos inherentes a las actividades en materia de salud, otros riesgos peculiares características del trabajo en este nivel de atención y que muchos no son conscientes de los riesgos laborales a que están expuestos. Conclusión: hay un número limitado de estudios sobre este tema, es necesario ampliar la discusión de los riesgos laborales, identificando las características de la atención básica de salud, fortalecer las acciones de vigilancia de la salud de los trabajadores y revisar la legislación que regulan el sector para proporcionar información que lleva a los profesionales a reflexionar sobre su auto-cuidado, así como la demanda de mejores condiciones de trabajo.
\end{abstract}

Palabras clave: Los riesgos profesionales; Atención sanitaria básica; Atención primaria de salud; Salud familiar; las enfermedades profesionales.

\title{
INTRODUÇÃO
}

Os trabalhadores estão constantemente expostos aos riscos ocupacionais em seu ambiente laboral. Os riscos ocupacionais podem ser compreendidos como uma ou mais condições do processo de trabalho com o potencial necessário para causar danos, rompendo com o equilíbrio físico, mental e social dos trabalhadores. ${ }^{(1,2)}$ No entanto, a exposição aos riscos ocupacionais não está necessariamente associada às doenças ocupacionais e aos acidentes de trabalho, pois isso depende do tempo ou da duração da exposição, das práticas e dos hábitos laborais, assim como da susceptibilidade individual do trabalhador. ${ }^{(1,3)}$

Observa-se que a legislação que regulamenta a saúde do trabalhador apresenta classificações referentes aos riscos ocupacionais que se complementam, demonstrando uma evolução no reconhecimento destes riscos no processo dinâmico do trabalho. O Ministério do Trabalho e Emprego classifica os riscos ocupacionais na Norma Regulamentadora 9 (NR) como riscos físicos, químicos e biológicos, ${ }^{(4)}$ e insere na NR 5, em seu Anexo IV que trata sobre o mapa de riscos, a ocorrência dos riscos ergonômicos e de acidentes. ${ }^{(5)}$

Posteriormente, com o reconhecimento das mudanças ocorridas na organização dos processos de trabalho e nas novas formas de adoecimento delas resultantes, incluiu-se a avaliação dos riscos psicossociais entre os riscos ocupacionais. ${ }^{(6,7)}$ 
No setor saúde, devido às peculiaridades das atividades desenvolvidas, os profissionais estão expostos aos riscos físicos, químicos, biológicos, ergonômicos (mecânicos), psicossociais e de acidente. Essa exposição contínua e múltipla pode promover o adoecimento dos trabalhadores e acarretar prejuízos às instituições de saúde empregadoras e as instituições governamentais, podendo interferir na qualidade da assistência prestada aos usuários, ${ }^{(8,9)}$ uma vez que, o estado de saúde do trabalhador interfere diretamente no desenvolvimento das suas atividades laborais.

A Atenção Básica, que se constitui como porta de entrada preferencial para o sistema da saúde brasileiro, sobretudo, após a implantação da Estratégia Saúde da Família (ESF), emprega atualmente grande parte dos profissionais do setor saúde. ${ }^{(1,8)}$ Estes profissionais estão expostos a alguns riscos que os diferenciam dos profissionais que atuam na área hospitalar, e, por isso, os torna específicos. ${ }^{(8,9)}$

Estudos revelam que os profissionais de saúde, especificamente os da Atenção Básica, não identificam os riscos no ambiente laboral e a exposição aos mesmos nas atividades que executam. $^{(1,9,10,11,12,13)}$ Desta forma, a prevenção se constitui na estratégia mais eficaz para evitar agravos à saúde e para tanto, faz-se necessário que as empresas estruturem consistentes padrões de monitoramento e controle da segurança, saúde e meio ambiente, bem como, que os trabalhadores estejam capacitados para reconhecer os riscos ocupacionais aos quais estão expostos e as medidas preventivas e de proteção individual e coletiva a serem adotadas frente aos mesmos.

Tendo em vista a expansão dos profissionais que atuam na Atenção Básica e a escassez de estudos publicados sobre a temática, entende-se a importância de discutir os riscos a que estão expostos estes trabalhadores, proporcionando informações que levem os profissionais a refletirem sobre o seu auto-cuidado, bem como reivindicarem por melhores condições de trabalho.

Desta forma, este trabalho tem como objetivo analisar os riscos ocupacionais a que estão expostos os profissionais atuantes na Atenção Básica de Saúde.

\section{MÉTODO}

Para elaboração do presente estudo, realizou-se uma revisão bibliográfica na base de dados da Biblioteca Virtual de Saúde (BVS), considerada uma das principais bases da área de 
saúde, a fim de identificar os riscos ocupacionais a que estão expostos os profissionais atuantes na Atenção Básica.

No levantamento dos artigos, foram utilizados, a princípio, os Descritores em Ciências da Saúde (DeCS): riscos ocupacionais, atenção básica, atenção básica de saúde, atenção básica à saúde, atenção primária à saúde, programa saúde da família, saúde da família e doenças ocupacionais. A utilização de um número elevado de descritores se justifica pelo fato de não haver um consenso na literatura sobre a terminologia mais adequada a representar o primeiro nível de atenção do setor saúde nos distintos países.

Após leitura dos resumos dos artigos encontrados foram selecionados apenas oito que respondiam ao objetivo do estudo. Por acreditar que essa amostra não era suficiente para embasar o estudo, foi realizado um segundo levantamento a partir das seguintes palavraschaves: riscos ocupacionais, atenção básica, atenção primária, atenção primária à saúde, programa saúde da família, estratégia saúde da família, doenças ocupacionais e saúde ocupacional.

Foram selecionados artigos científicos publicados no período de 2000 a 2011 que se encontravam no idioma português e espanhol. Considerou-se para elaboração deste trabalho os riscos ocupacionais físicos, químicos, biológico, ergonômicos, de acidente e psicossociais. Posteriormente foi realizada uma leitura sistemática dos resumos dos trabalhos encontrados e quando os mesmos se relacionavam com o objetivo do estudo e apresentavam texto disponível na íntegra foram selecionados para uma leitura aprofundada.

Foram excluídos da pesquisa os artigos que trataram dos riscos ocupacionais a que estão expostos os profissionais de saúde não inseridos na Atenção Básica, teses, dissertações, monografias e artigos em outros idiomas que não fossem o português e o espanhol.

Em seguida, procedeu-se ao fichamento dos 20 artigos selecionados em uma tabela considerando o título do artigo; autores; revista, ano de publicação e local do estudo; palavraschave e descritores, tipo de metodologia utilizada e riscos ocupacionais abordados no artigo.

Posteriormente, os artigos foram classificados por categoria de risco (físicos, químicos, biológicos, ergonômicos, psicossociais e de acidentes). Os dados foram analisados com base em literatura específica e na legislação referente à saúde do trabalhador. 


\section{RESULTADOS E DISCUSSÃO}

Dos 20 estudos selecionados para análise, 18 (90\%) foram artigos originais, os quais utilizaram diferentes metodologias para coleta dos dados e dois (10\%) eram artigos de revisão. Destes, $17(85 \%)$ apresentavam-se no idioma português e foram desenvolvidos no Brasil; os três estudos restantes (15\%) estavam no idioma espanhol e foram desenvolvidos no México (Guadalajara) e no Chile (Santiago), ambos apresentando sistema universal de saúde, semelhante ao Brasil.

A maioria da produção científica esteve concentrada nos últimos cinco anos, $80 \%$ dos artigos, caracterinzando-se em estudos recentes e apenas uma das publicações ocorreu no ano de 2001.

No que se refere à população estudada, oito artigos (40\%) utilizaram como amostra o conjunto de profissionais que atuam nas Unidades Básicas de Saúde, quatro (20\%) utilizaram todos os profissionais atuantes na Estratégia Saúde da Família, quatro (20\%) foram especifícos da categoria de enfermeiras; três (15\%) foram específicos para os Agentes Comunitários de Saúde (ACS) e um artigo (5\%) para a categoria médica.

Merece destaque o fato de não haver encontrado nenhum estudo que abordasse exclusivamente as demais categorias da saúde, integrantes da equipe multiprofissional (dentista, nutricionista, assistente social, entre outros), atuantes na Atenção Básica, ao que pode-se inferir que se deva ao fato da Saúde da Família ser sido inserida como estratégia prioritária da Atenção Básica e incorporar apenas na sua equipe médicos, enfermeiros, ACS e auxiliares de enfermagem. Os odontólogos, apesar de terem sido incorporados à ESF e estarem constantemente expostos aos riscos ocupacionais, não foram ainda objeto de estudos referentes a essa temática na Atenção Básica.

Todos os riscos (físico, químico, biológico, ergonômico, de acidente e psicossocial) elegíveis pelas autoras foram abordados nos diversos estudos encontrados, no entanto, nem todos os artigos discorreram sobre os seis tipos de risco concomitantemente.

Os riscos psicossociais foram exclusivamente abordados em oito (40\%) artigos. Cinco estudos (25\%) discorreram sobre mais de três tipos de riscos classificados com base nas normas regulamentadoras que regem a saúde do trabalhador, seguidos de quatro (20\%) que abordaram apenas o risco biológico, dois (10\%) apresentaram os riscos psicossociais e violência e apenas um estudo (5\%) abordou exclusivamente o risco ergonômico. 
A análise dos artigos proporcionou identificar que a maioria dos autores dos estudos descreveu os diversos tipos de riscos ocupacionais (físico, químico, biológico, ergonômico, de acidente e psicossocial) sem, no entanto, utilizarem a nomenclatura adotada pelas Normas Regulamentadoras referentes à saúde do trabalhador. Com exceção dos estudos desenvolvidos em outros países, que não são regidos pelas normas brasileiras, esperava-se que os autores se baseassem nesta legislação para classificar tais riscos.

Os riscos biológicos, entendidos como a exposição aos agentes biológicos bactérias, fungos, bacilos, parasitas, protozoários, vírus, entre outros, ${ }^{(4)}$ geralmente, são os mais estudados em relação aos profissionais de saúde, pois estão diretamente associados às práticas desenvolvidas por estes trabalhadores. No entanto, este tipo de risco é mais comumente abordado apenas para os profissionais que atuam na área hospitalar. ${ }^{(8,10,14)}$

$\mathrm{Na}$ Atenção Básica, as atividades desenvolvidas pelas diversas categorias profissionais que envolvem o atendimento direto ao usário tanto no serviço quanto nos espaços da comunidade os tornam expostos a esse tipo de risco. Alguns estudos revelaram que acidentes com perfurocortantes ocorreram com técnicos e auxiliares de enfermagem, enfermeiras, cirurgiões-dentista e médicos durante os procedimentos de punção venosa, teste de glicemia, administração de medicamentos, realização de curativos e suturas, procedimentos odontológicos, descarte de material e administração de vacinas. ${ }^{(1,8,9,10,13,14,15,16)}$ Além disso, houve exposição a material biológico durante a realização do exame papanicolau, durante as consulta de tuberculose e hanseníase, dentre outras doenças transmissíveis. ${ }^{(14,17,18)}$ e existem estudos que apontaram a associação do risco biológico ao trabalho desempenhado pelos Agentes Comunitários de Saúde, sobretudo às doenças infecciosas. ${ }^{(14,17,18)}$

Vale destacar que em um dos estudos, os profissionais entrevistados referiram algumas doenças parasitárias como recorrente em sua prática, ainda que esta enfermidade não esteja contemplada na relação das doenças relacionadas ao trabalho. ${ }^{(10)}$

Outro aspecto importante a ser observado é a situação vacinal dos Agentes Comunitários de Saúde, ainda pouco abordada na atenção primária, assim como a dos outros profissionais que vêm sendo incorporados a ESF, como, por exemplo, os auxiliares de consultório dentário e os técnicos em higiene dental, membros das equipes de saúde bucal. ${ }^{(14,18)}$

Os riscos físicos são definidos como a exposição às diversas formas de energia a que possam estar expostos os trabalhadores, tais como: ruído, vibrações, pressões anormais, temperaturas extremas, radiações ionizantes, radiações não ionizantes, bem como o infra-som 
e o ultra-som. ${ }^{(4)}$ Temperatura ambiental desconfortável, nível de ruído incômodo e irritante, exposição à iluminação precária, a falta de arejamento nos consultórios, as instalações elétricas inadequadas, à exposição ao sol, ao calor, a chuva e ao frio, principalmente durante a realização das visitas domiciliares foram os principais riscos físicos apontados pelos profissionais da Atenção Básica nos diferentes estudos. ${ }^{(1,9,13,18,19)}$ Diante desta realidade, sinaliza-se a importância da implementação de medidas de manutenção preventiva nos equipamentos e na infraestrutura das unidades de saúde, bem como a oferta de equipamentos de proteção individual (boné, protetor solar, capas de chuva, botas, entre outros) que protejam o trabalhador da exposição à alguns destes riscos.

Os riscos químicos não são tão comuns na Atenção Básica quanto o são no ambiente hospitalar. No entanto, agressões a pele devido ao uso freqüente de sabão e álcool, bem como à utilização de luvas de procedimento, à administração de medicamentos e soluções, o manuseio de uma variedade grande de substâncias químicas, tais como desinfetantes, desencrostantes ou esterilizantes, anestésicos e antissépticos e a exposição à fumaça do cigarro e de veículos durante as práticas desenvolvidas na comunidade são alguns dos riscos químicos identificados nos estudos. ${ }^{(1,9,18)}$ Desta forma, entende-se como risco químico a exposição aos seguintes agentes químicos: substâncias, compostos ou produtos que possam penetrar no organismo pela via respiratória, nas formas de poeiras, fumos, névoas, neblinas, gases ou vapores, ou que, pela natureza da atividade de exposição, possam ter contato ou ser absorvidos pelo organismo através da pele ou por ingestão. ${ }^{(4)}$

É necessário reforçar entre estes trabalhadores a importância da adoção das medidas de proteção padrão, uma vez que, muitos destes riscos são inerentes ao processo de trabalho com intervenções em saúde, não havendo a possibilidade de sanar a exposição, apenas de minimizá-la.

Os aspectos referentes aos riscos ergonômicos são ainda incipientes na Atenção Básica, embora sabe-se que a inadequação ergonômica dos postos de trabalho constitui um problema importante para os profissionais, uma vez que apresenta reflexos na saúde e produtividade dos mesmos. ${ }^{(20)}$ Os estudos revelaram que durante a realização das atividades laborais, os profissionais estão expostos a estes tipos de risco, tais como: postura inadequada para administração de vacinas, medicamentos e realização de procedimentos, mobiliários inadequados e sobrecarga de peso durante as atividades..$^{(1,9,1318,20,21,23)}$ Nenhum dos estudos selecionados fizeram referência a exposição ao risco ergonômico entre os odontólogos, entretanto, esta categoria merece destaque devido às características das atividades destes 
profissionais, que trabalham constantemente em posturas inadequadas, sem períodos de repouso e sob forte tensão emocional. ${ }^{(21)}$

O risco de acidente, referido no anexo IV da NR 5, é bem perceptível na prática laboral dos profissionais atuantes na Atenção Básica. Alguns riscos de acidentes foram identificados nos estudos, como: quedas diante das adversidades do solo; exposição à picada de animais peçonhentos e mordedura de cães; acidentes provocados por materiais perfurocortantes; risco de choque elétrico por contato com fiação inadequada e cercas elétricas; visitas a moradias construídas em locais sob risco de desabamentos; entre outros. $^{(1,10,13,18)}$

Além disso, os profissionais que atuam na Estratégia Saúde da Família estão expostos, além dos riscos mencionados, ao risco de acidente de trajeto, ${ }^{(1,13)}$ pois necessitam se deslocar da sua residência para as unidades de saúde, normalmente, localizadas em áreas periféricas e longínquas com o objetivo de atender a uma parcela mais carente da população, respeitando o principio da equidade preconizado pelo Sistema Único de Saúde (SUS). ${ }^{(26)}$

O risco psicossocial foi abordado em 15 trabalhos (75\%), sendo que oito estudos (40\%) analisaram exclusivamente estes riscos e, além disso, foi o risco mais referido pelos profissionais nesses estudos. A Atenção Básica, sobretudo a ESF, orienta-se pelos princípios da universalidade, acessibilidade, vínculo e continuidade, responsabilização e humanização. ${ }^{(22)}$ As características do processo de trabalho dos profissionais desse nível de atenção têm aumentado a sobrecarga psíquica dos trabalhadores, pois as condições de trabalho, associada ao tipo de relação que são estabelecidas com os usuários e com a comunidade, não favorecem o alcance efetivo desses princípios. ${ }^{(10,25,26,29)}$

Os estudos apontaram um número significativo de riscos psicossociais, dentre eles: estresse; sobrecarga mental; sobrecarga de atividades; rígido controle do tempo; forma como o setor é organizado; falta de materiais e equipamentos adequados; conflitos nos relacionamentos entre os membros da equipe; pressão da própria clientela, que não entende essa nova proposta de assistência à saúde e ainda prefere os modelos curativos ao enfoque preventivo; ansiedade; demandas sociais da população e violência. $(1,9,10,13,18,21,23,24,25,26,27,28,29,30,31,32)$

Destaca-se que dois estudos ${ }^{(1,18)}$ utilizaram a nomenclatura risco ergonômico para se referirem aos riscos que apresentam características de riscos psicossociais. Observa-se, portanto, a necessidade de uma padronização dos conceitos e classificações destes riscos. 
A violência foi mencionada em vários estudos como um importante risco psicossocial. $^{(10,18,19,26,28,30)}$ Esta constatação pode ser justificada pelo fato de as equipes de saúde da Atenção Básica, especialmente, as equipes de Saúde da Família serem implantadas em áreas periféricas com importantes índices de violência relacionados à presença de narcotráfico. $^{(13,18,26)}$

Outro risco psicossocial relevante é a precarização do trabalho, que interfere nas condições de saúde do trabalhador, destacando a precariedade dos vínculos empregatícios no setor público de saúde o qual faz com que o trabalhador não possa usufruir dos direitos profissionais. $^{(1,14,15,25)}$

A relação entre exposição ocupacional aos riscos e a morbidade, propriamente dita, não se caracteriza como uma relação de causa e efeito, pois depende da freqüência e duração da exposição, do tipo de prática desenvolvida e das características individuais do trabalhador. ${ }^{(1,3)}$ No entanto, alguns estudos apontaram a relação entre os riscos psicossociais e diferentes morbidades que acometeram os profissionais da Atenção Básica. (21,23,24,25,26, 27,28)

Assim, percebe-se que os profissionais atuantes na Atenção Básica além de estarem expostos aos riscos tradicionalmente já discutidos e conhecidos como comuns aos profissionais da área da saúde, estão expostos também a alguns riscos peculiares as características deste nível de atenção, sobretudo na Estratégia Saúde da Família, considerada como porta de entrada preferencial ao sistema de saúde no Brasil. Soma-se a isso o fato de que muitos profissionais não têm consciência dos riscos ocupacionais a que estão expostos. $^{(1,9,10,11,12,13,18,21)}$

Frente a vulnerabilidade ao adoecimento e aos acidentes de trabalho entre os profissionais de saúde da Atenção Básica, onde inclui-se os profissionais administrativos e de limpeza, os estudos apontam para a necessidade de implementação de uma política ampla de gerenciamento de recursos humanos, sobretudo para os profissionais que apresentam vínculos empregatícios precários, considerando os riscos e a complexidade inerente às atividades desenvolvidas por esses profissionais. ${ }^{(15,18)}$

Faz-se necessário a implementação de ações voltadas para a imunização de todos os profissionais da atenção primária, com posterior verificação do estado vacinal, bem como ações de educação permanente para os profisssionais de todos os níveis e funções, incluindo como tema prioritário a utilização dos equipamentos de proteção individual. ${ }^{(14,15,16,18,19)}$ 


\section{CONCLUSÃO}

Este estudo permitiu identificar que há um número limitado de trabalhos referentes à exposição dos profissionais de saúde atuantes na Atenção Básica aos riscos ocupacionais, fato já mencionado por outros autores. Possibilitou também discutir os riscos ocupacionais aos quais estes trabalhadores estão expostos, como os riscos químicos, físicos, biológicos, ergonômicos, de acidente e psicossocial.

Tendo em vista que os recursos humanos assumem o papel central na Atenção Básica, é fundamental garantir a saúde do trabalhador do setor saúde, focalizando a qualidade dos serviços prestados à população numa relação direta com a satisfação do direito à saúde e segurança no trabalho.

Sugere-se que os órgãos responsáveis pela Saúde do Trabalhador ao reconhecer as especificidades da prática profissional desenvolvida neste nível de atenção e seus respectivos riscos, as incorpore nas normatizações que regulamentam o setor. Além disso, que as Normas Regulamentadoras sejam efetivamente aplicadas nos serviços públicos de saúde e a seus trabalhadores.

Logo, destaca-se a importância de fortalecer a Vigilância em Saúde do Trabalhador através dos Centros de Referência em saúde do Trabalhador (CEREST), cujas ações estão centradas na relação da saúde com o ambiente e os processos de trabalho e nesta com a assistência, baseadas nos princípios da vigilância para a melhoria das condições de vida e saúde dos trabalhadores. ${ }^{(34)}$

Faz-se necessário ampliar a percepção dos contextos que asseguram a promoção da saúde destes profissionais, bem como a discussão recorrente sobre os riscos aos quais estão expostos estes trabalhadores, proporcionando informações que levem os profissionais a refletirem sobre o seu auto-cuidado, bem como reivindicarem por melhores condições de trabalho.

\section{REFERÊNCIAS}

1. Bessa MEP, Almeida MI de, Araújo MFM, Silva MJ da. Riscos ocupacionais do enfermeiro atuante na Estratégia Saúde da Família. Rev. enferm. UERJ 2010; 18(4): 644-49.

2. Brasil. Ministério da Saúde. Secretaria de Assistência à saúde. Departamento de normas técnicas. Segurança no ambiente hospitalar. Brasília; 1995. 
3. Xelegati R, Robazzi MLCC. Riscos químicos a que estão submetidos os trabalhadores de enfermagem: uma revisão de literatura. Rev Latino-am Enfermagem 2003; 11(3):350-6.

4. Brasil, Ministério do Trabalho e Emprego. Norma Regulamentadora 9: programa de prevenção de riscos ambientais. Portaria SSST no 25 de 29/12/1994. [acesso em 2011 jun 10]. Disponível em: http://www.mte.gov.br/legislacao/normas_regulamentadoras/nr_9

5. Brasil. Ministério do Trabalho e Emprego. Norma Regulamentadora - NR 5: comissão interna de prevenção de acidentes. [acesso em 2011 jun 10]. Disponível em: http://www.mte.gov.br/legislação/normas_regulamentadoras/nr_5

6. Organization Mundial de La Saúde. Vigilanicia Del madio y de las condiciones de salud em los programas de higiene del trabajo. Genebra; 1973. (Série Informes Técnicos).

7. Ministério da Saúde do Brasil. Organização Pan-Americana da Saúde no Brasil. Doenças relacionadas ao trabalho: manual de procedimentos para os serviços de saúde. Brasília, DF: OPAS/OMS; 2001.

8. Chiodi MB, Marziale MHP, Robazzi MLCC. Acidentes de trabalho com material biológico entre trabalhadores de unidades de saúde pública. Rev Latino-am Enfermagem. 2007; 15(4).

9. Chiodi MB, Marziale MHP. Riscos ocupacionais para trabalhadores de unidades básicas de saúde: revisão bibliográfica. Acta Paul Enferm. 2006; 19(2):212-17.

10. Cezar-Vaz MR et al. Percepção do risco no trabalho em saúde da família: estudo com trabalhadores no sul do Brasil. Rev Latino-am Enfermagem 2009; 17(6).

11. Silva RCG, Felli VEA. Um estudo comparativo sobre a identificação dos riscos ocupacionais por trabalhadores de enfermagem de duas unidades básicas de saúde do município de São Paulo. Rev Esc Enferm USP 2002; 36(1): 18-24.

12. Farias SNP de, Zeitoune RCG. Riscos no trabalho de enfermagem em um centro municipal de saúde. R Enferm UERJ 2005; (13):167-74.

13. Nunes MBG et al. Riscos ocupacionais dos enfermeiros atuantes na Atenção à Saúde da Família. Rev. enferm. UERJ 2010; 18(2): 204-09.

14 Alcântara CCS et al. Riscos ocupacionais na atenção primária à saúde: susceptibilidade às doenças imunopreveníveis dos profissionais que atuam em uma unidade básica de saúde de Fortaleza, Ceará - 2003. Rev. APS 2005; 8(2): 143-150.

15. Garcia LP, Facchini LA. Vacinação contra a hepatite B entre trabalhadores da atenção básica à saúde. Cad. Saúde Pública. 2008; 24(5):1130-1140.

16. López JC et al. Accidentabilidad laboral con productos biológicos en profesionales sanitarios de Atención Primaria de Guadalajara (1994-2000). Rev. Salud Ambient. 2001;1(2): $87-91$. 
17. Rodrigues PM et al. Infecção por Mycobacterium tuberculosis entre agentes comunitários de saúde que atuam no controle da TB. J Bras Pneumol. 2009; 35(4):351-358.

18. Nascimento GM, David HMSL. Avaliação de riscos no trabalho dos agentes comunitários de saúde: um processo participativo. Rev. enferm UERJ. 2008; 16(4):550-6.

19. Simões AR. O agente comunitário de saúde na equipe de saúde da família: fatores de sobrecarga de trabalho e estresse. R. Saúde Públ. 2009; 2 (1): 6-21.

20. Hass GG et al. Condições ergonômicas em uma unidade básica de saúde recentemente informatizada de Florianópolis - SC. ACM arq. catarin. med. 2008; 37(4): 27-31.

21. Araújo MA, Paula MVQ. Ler/Dort: Um Grave problema de saúde pública que acomete os Cirurgiões-Dentistas. Revista APS. 2003; 6(2): 87-93.

22. Brasil. Ministério da Saúde. Portaria MS/GM,648, 28/03/2006. Política Nacional de Atenção Básica. [acesso em 2011 jul 09]. Disponível em: http://dtr2001.saude.gov.br/sas/PORTARIAS/Port2006/GM/GM-648.htm

23. Tomasi E. Condições de trabalho e automedicação em profissionais da rede básica de saúde da zona urbana de Pelotas, RS. Rev Bras Epidemiol. 2007; 10(1): 6674.

24. Beltrán CA, Moreno MP. Factores psicosociales asociados a patologías laborales en médicos de nivel primario de atención en Guadalajara, México. Rev Med Urug. 2007; 23(4): 251-9.

25. Santos VC, Soares CB, Campos CMS. A relação trabalho-saúde de enfermeiros do PSF no município de São Paulo. Rev Esc Enferm USP. 2007; 41(Esp): 777-81.

26. Lancman $S$ et al. Repercussões da violência na saúde mental de trabalhadores do Programa Saúde da Família. Rev Saúde Pública. 2009;43(4):682-8.

27. Camelo SHH, Angerami ELS. Riscos psicossociais relacionados ao trabalho das equipes de saúde da família: percepções dos profissionais. R Enferm UERJ, 2007; 15(4):502-7.

28. Wai MFP, Carvalho AMP. O trabalho do agente comunitário de saúde: fatores de sobrecarga e estratégias de enfrentamento. Rev. enferm. UERJ. 2009; 17(4):563-8.

29. Braga LC et al. Condições de trabalho e transtornos mentais comuns em trabalhadores da rede básica de saúde de Botucatu (SP). Ciênc. saúde coletiva. 2010; 15(Supl. 1):1585-1596.

30. Kaiser DE, Bianchi F. A violência e os profissionais da saúde na atenção primária. Rev Gaúcha Enferm. 2008; 29(3):362-6.

31. Feliciano KVO, KMH, Sarinho SW. Superposição de atribuições e autonomia técnica entre enfermeiras da Estratégia Saúde da Família. Rev. Saúde Pública. 2010; 44(3): 520-7. 
32. Guic SE, Mora OP, Rey CR, Robles GA. Estrés organizacional y salud en funcionarios de centros de atención primaria de una comuna de Santiago. Rev. méd. Chile. 2006; 134(4): 447455 .

33. Martines WRV, Chaves EC. Vulnerabilidade e sofrimento no trabalho do agente comunitário de saúde no Programa de Saúde da Família. Rev. esc. enferm. USP. 2007; 41(3): 426-33.

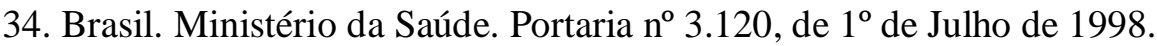

